\title{
la carretera de hormigón
}

Hemos creido conveniente, por su interés, el dar a conocer el presente articulo aparecido en la revista "La Route en Béton".

\section{Las nuevas normas suizas para firmes de hormigón. Edición 1961}

La Unión Sutza de Profestonales de la Carretera (VSs) había publicado en 1945 y 1946 las antiguas "Directrices para la construcción de carreteras de hormigón" y las "Condiciones especiales para obras de carreteras", de las cuales la parte $\mathrm{V}$ del tercer capitulo se refería a los firmes de hormigón. Desde entonces, investigaciones y experiencias han dado lugar a modiflcaciones a veces fundamentales en lo que se reflere a la construcción de la carretera $y$, concretamente, a la ejecuclón de los pavimentos de hormigón. Durante los últimos quince años las cargas y densidad de tráfico han aumentado considerablemente, al mismo tiempo que han aparecido nuevas máquinas que permiten la construcción más racional de mejores cimientos y firmes.

Las nuevas normas establecidas por la Comisión de Flrmes de Vss tienen en cuenta los nuevos conocimlentos. Fstas normas están contenidas en las sigulentes hojas relativas a las carreteras de hormigón:

SNV 40462 Principios de construcción.

40462 Exigencias de calidad para los materiales.

40465 Prescripclones de ejecución.

40468 Condiciones de recepción del pavimento.

40470 Obturación de juntas.

Prescripclones de ejecución.

Nos proponemos comentar aquí los puntos principales de estas nuevas normas, dar expllcaciones complementarias e indicar las modiflcaclones introducidas en relación con las normas anttguas. Beguiremos para ello el orden normal de operaciones de la construcción y no el de numeración, blen entendido que los presentes comentarios no sustituyen en nada a dichas normas.

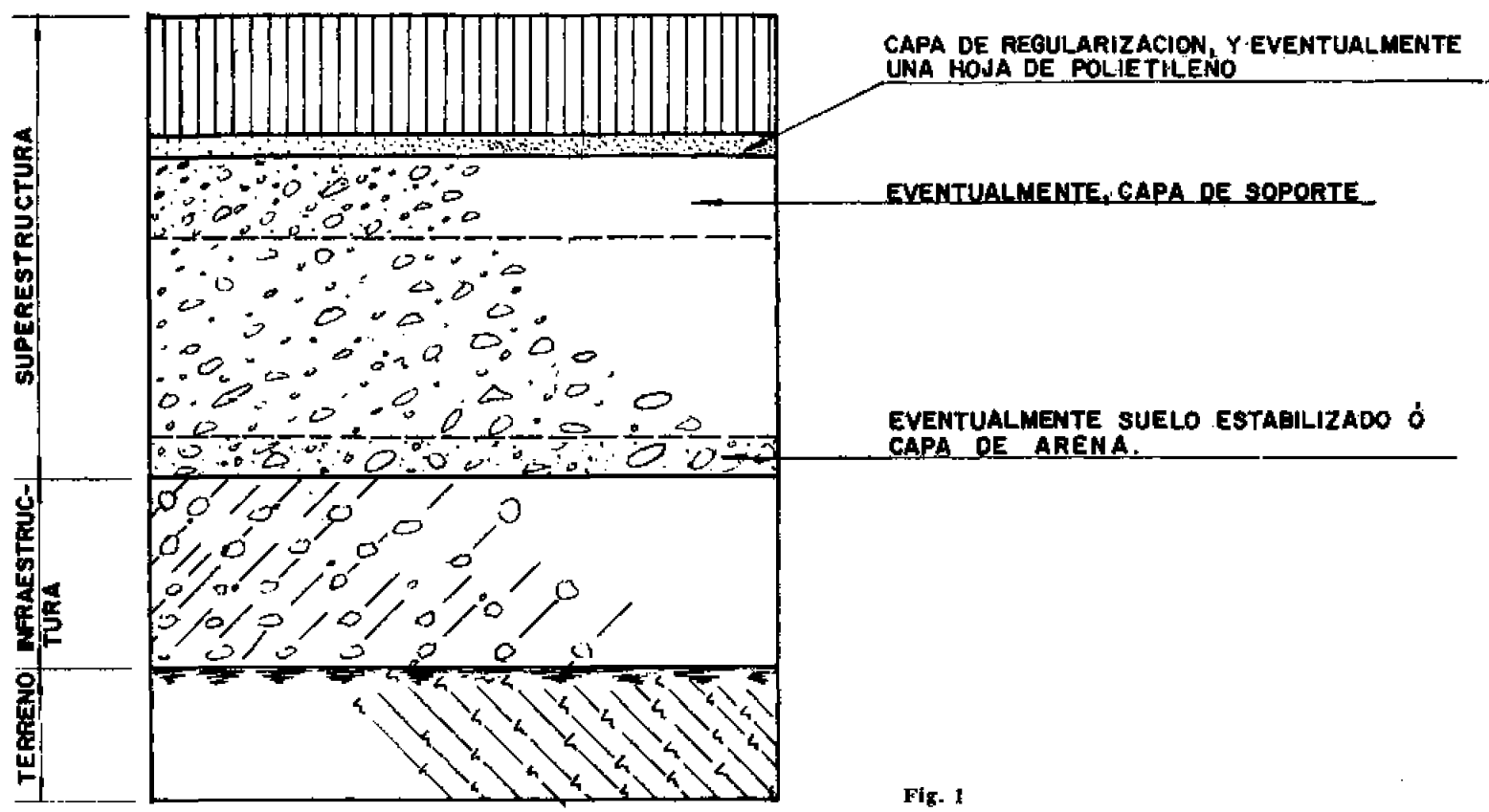




\section{Cimiento}

El objeto de la norma 40325 es determinar las dimensiones y calidad de los cimientos, en tanto que la 40372 flja las condiciones de compactación.

En principio, el firme de hormigón reposa directamente sobre el cimlento terminado, a veces con una capa de regularización. En ciertos casos, se utilizan con éxlto hojas de plástico desenrolladas sobre el cimiento, con el fin de evitar que el agua que pudiera encontrarse en éste suba al hormigón por efecto de la vibración, o que el mortero descienda a la capa de soporte. Cuando el cimiento está bien terminado y cerrado, puede prescindirse de la capa de regularización.

La superficie del cimiento no debe presentar desigualdades superiores a $2 \mathrm{~cm}$ medidas con un reglón de 4 metros.

Comoquiera que las losas de hormigón proporcionan un amplio reparto de cargas sobre el terreno, la capacidad resistente, $\mathrm{ME}=1.000 \mathrm{~kg} / \mathrm{cm}^{2}$, prescrita por la norma 40372 en la superflcie del cimiento, se puede reducir a $500 \mathrm{~kg} / \mathrm{cm}^{2}$ para los firmes que soportan un tráfico ligero o medio. Lo que importa, esencialmente, es la regularidad de esta capacidad.

En ciertos casos especiales se puede, también, disponer una capa de soporte bajo el flrme de hormigón (grava machacada, eventualmente con riego de penetración o revestimientos asfálticos). Puede presentarse este caso cuando la construcción del pavimento deba ser demorada, con objeto de dar lugar a que se produzcan eventuales deformaciones irregulares en la carretera para evitar las cuales no haya sido posible adoptar una medida más adecuada.

Las nuevas normas relativas al cimiento son, por lo tanto, sensiblemente diferentes de las antiguas que, sobre un suelo de mala sustentación, no preveían más que una simple capa de grava ligeramente apisonada.

\section{Tipos de pavimentos}

Las normas hacen una distinción entre el pavimento normal de una o dos capas y los pavimentos especiales, tales como firmes de hormigón pretensado de losa doble o de elementos prefabricados.

En Suiza se construyen firmes de dos capas, fundamentalmente por razones económfcas. La capa inferior no precisa más que de grava rodada $y$ barata porque se encuentra abundantemente, mientras que para la capa superior más delgada se utilizan, generalmente, gravas duras procedentes đe machaqueo. De esta manera se evita que las piedras frecuentemente agrietadas que aparecen en Ias graveras de la meseta vayan a parar a la superficie del firme, donde serían destruídas por las heladas.

El firme de doble losa es necesarto cuando el espesor total pasa de los $30 \mathrm{~cm}$, porque el flrme deba resistir cargas excepcionalmente fuertes. Las dos capas se compactan mejor separadamente por medio de vigas vibrantes, que si se trabajan a pleno espesor. Por otra parte, las tenstones que provocan en cada una de ellas las diferencias de temperatura en tre la superflcie superior e inferior son menores que en una losa única de mayor espesor. Los flrmes de doble losa se utilizan también para reforzar los firmes demasiado débiles. Mencionemos, por ejemplo, el refuerzo reciente de la antigua pista de cointrin (con y sin capas de separación) y el tramo de ensayo de Schottikon sobre la carretera WinterthurSt. Gall, sin capa de separación.

Solamente se ha tenido ocasión de construlr en Suiza flrmes de elementos prefabricados por vía de ensayo en un pequeño camino rural.

\section{Espesores de firmes}

Las normas fljan los espesores mínimos sigulentes:

$\begin{array}{lr}\text { a) Pistas para bicicletas y aceras } & 8 \mathrm{~cm} \text {. } \\ \text { b) Carreteras de urbanización y caminos rurales } & 14 \mathrm{~cm} \text {. } \\ \text { c) Carreteras con tráflco medio } & 16 \mathrm{~cm} \text {. } \\ \text { d) Carreteras con tráflco pesado } & 18 \mathrm{~cm} \text {. } \\ \text { e) Carreteras con tráflco muy pesado } & 20 \mathrm{~cm}\end{array}$




\section{Dimensiones de las losas}

En carreteras se aconseja que la anchura de las losas no rebase los $4,50 \mathrm{~m}$ y su longitud quede comprendida entre los 6 y 12 metros. La longitud menor, es decir, los 6 metros, debe emplearse en zonas donde el valor de la sustentacion sea variable de uno a otro perfil, en las curvas de radio pequeño y en las transiciones de desmonte a terraplén.

En los firmes que deben soportar cargas muy elevadas, como pistas de aviación, se debe tender, preferentemente, a paneles cuadrados.

\section{Armodura}

Todos los firmes de hormigón deben disponer de una armadura, a excepción de los que no soportan más que un tráflco pequefio. Para los casos normales, las nuevas normas confirman el uso, adoptado desde hace tiempo en suiza, de una armadura de bordes de losa compliestas por mallas metálicas, coiocadas entre las dos capas de hormigón inferior y superior, y barras de acero II, colocadas en la parte inferior.

Sobre un terreno que se comprima destgualmente, hace falta reforzar esta armadura cubriendo toda la superficie con un emparrillado de acero de, por lo menos, $1,7 \mathrm{~kg} / \mathrm{m}^{2}$.

Las normas establecen nuevas exigencias de calidad para los emparrillados de acero. Se fija, para los alambres, el límite aparente de elasticidad, el alargamiento de rotura y resistencia al doblado repetido; para las soldaduras se determina la dureza vickers, la resistencia a la tracción, la resistencia a flexión, el esfuerzo de desgarramiento, y se determina el ensayo a esfuerzos repetidos.

Fiy. 2.-Armadura normal de tha losa.

1. Emparrillado de acero a lo lario de tos bordes, colocado entre las capas inferiar y superior.-2. Armadura de bordes de la capa inferior $\phi$ io o $12 \mathrm{~mm}-3$. Pasactores de redondo longitudinates (acero corrugado.

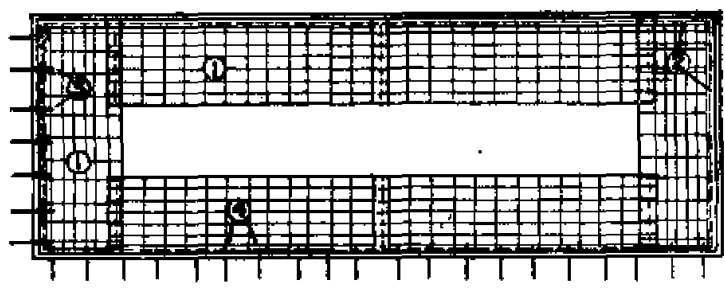

\section{Juntos}

Las nuevas normas tienen en cuenta los grandes progresos en la constitución de juntas, realizados en el curso de los últimos años. En las carreteras importantes, estas juntas deben ser desde ahora realizadas mecánicamente para evitar todas las desigualdades, desagradables a los usuarios, que pudiera dejar el trabajo manual.

En la mayoria de las carreteras, las juntas longitudinales son aserradas en el hormigón endurecido. En principio están provistas de barras de unión y, a veces, para las losas de espesor superior a los 18 centimetros, de un dispositivo de enlace en el hormigón.

En general, el corte superior de las juntas fransversales de contracción o dilatactón se realiza mecánicamente.

En las carreteras de montaña muy sinuosas y con curvas de poco radio, así como en los firmes de menos de $18 \mathrm{~cm}$ de espesor, se disponen de juntas de dilatación cada 40 a $60 \mathrm{~m}$, como se hacia antes. En los pavimentos de más espesor se han suprimido deflnitivamente las juntas de dilataclón, excepto en las curvas de débil radio y a la entrada y salida de zonas singulares tales como puentes. Las observaclones hechas en el extranjero, especialmente en los Estados UnIdos, confirman que los pavlmentos de clerto espesor pueden soportar, sin detrimento, la compresión debida a la dilatación térmica. La supresín de juntas de dílatación somete a las losas a un efecto de pretensado qúe, a pesar de no ser permanente, mejora las condiciones resistentes del pavim ento.

En las carreteras de tráfico pesado, las juntas de contracción y las longitudinales se proveen de pasadores y, además, de un dispositivo de transmisión de esfuerzos en el hormigón, constituído o por un machihembrado o por la interposición de una lámina ondulada de fibrocemento. En este caso, es decir, cuando se pone una tira de fibrocemento ondulado en la parte inferior de la losa, la junta toma la forma de una superficie conoidal, en la que el fibrocemento constituye la directriz inferior y el corte rectilfneo de la superficie es la directriz superior. Esta forma de junta constituye en sí un buen dispositivo de transmisión de cargas. 
Para los pasadores se utiliza acero de alta calidad cuyo límite aparente de elasticidad es superior a $50 \mathrm{~kg} / \mathrm{mm}^{2}$.

Las diferentes prescripciones que acabamos de comentar contribuyen a asegurar la perfecta transmisión de cargas en las juntas, de tal suerte que no se produzcan los famosos "escalones" que sufren las viejas autopistas alemanas.

\section{Aridos}

Deben recibirse en la obra por separado. En clertos casos especiales pueden ser mezclados por los proveedores que dispongan de instalaciones convenientes, pero en estos casos hay que tener cuidado de que no se segreguen en el transporte o en la descarga.

La composición granulométrica de la mezcla debe satisfacer a la norma SIA 162; puede tambièn basarse en la curva de Fuller. El módulo de flnura no debe separarse más del $5 \%$ del de la curva teórica. Se pueden también utilizar mezclas de granulometría discontinua, establecida determinando el volumen de huecos. Sin embargo, en Suiza, donde se suele utilizar materiales de río o de gravera, esta granulometría discontinua se aplica raramente.

Cualquiera que sea la curva granulométrica adoptada, las normas fijan que la proporclón de granos menores de 0,12 $\mathrm{mam}$ no debe pasar del $8 \%$ y Ia de los granos inferiores a $0,5 \mathrm{~mm}$ no rebasará el $20 \%$ de la fracción 0-8 mm. Además, para que el hormigón de la capa superior posea la rugosidad deseada, hay que añadirle una cantidad suficiente de grava dura 3-10 milímetros.

\section{Dosificación del cemento y resistencias del hormigón}

La proporción de cemento que fljan las normas que se comentan, queda igual que antes, o sea:

250 a $300 \mathrm{~kg} / \mathrm{m}^{3}$, para el hormigón de la capa inferior.

300 a $350 \mathrm{~kg} / \mathrm{m}^{3}$, para el hormigón de la capa superior.

Se han disminuido ligeramente las resistencias medias en relación con las de las antiguas normas, para aproximarlas un poco a las que exigen las normas SIA:

\begin{tabular}{|c|c|c|}
\hline \multirow[b]{2}{*}{ HORMIGON } & \multicolumn{2}{|c|}{ RESISTENCIAS MEDIAS } \\
\hline & $\underset{\text { Flexión }}{\left(\mathbf{K g} / \mathrm{cm}^{2}\right)}$ & 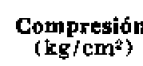 \\
\hline BH 250 & 40 & 260 \\
\hline BH 300 & 45 & 320 \\
\hline BH 350 & 50 & 380 \\
\hline
\end{tabular}

Estas resistencias pueden ser del $10 \%$ inferiores para el hormigón de aire ocluílo*.

Las normas exigen que la dosis de cemento sea adaptada a la calidad de los áridos y resistencias requeridas. Esta prescripción es importante en suiza $y$, especialmente, en clertas obras de montafia donde se utillza para el hormigón de la capa inferior áridos procedentes de graveras graniticas próximos a los nacimientos de los ríos y en los que, por lo tanto, el granito ha sido muy poco rodado. Sin más, que al aumentar la dosis de cemento, a menudo se pueden obtener resistencias suficientes en hormigones fabricados con estos granitos, como se ha comprobado en las colinas de Grisons, en el oberland bernés, en el Tessin y en la Leventine.

Esta grava granitica, que a menudo hay que utilizar con arena también granitica que contlene mica, da un excelente resultado para hormigones de la capa superior, pues jamás se pulfmenta por efecto đel tráfico. Damos como prueba de ello los firmes de hormigón sometldos desde hace más de veinte años a tráfico intenso en la rampa sur del Gothard.

- Normalmente conocido por hormigón aireado. 


\section{Adiciones}

Las normas mencionan la propiedad de los productos de adición llamados aireantes, que confteren a los hormigones una mayor resistencia a la acción de las sales empleadas para deshelar las calzadas. En Suiza se utilizan tales adiciones desde hace varios años en los hormigones de capa superior (contenido de alre: 3 a $4 \%$ ), aunque la resistencia a la compresión se disminuye ligeramente.

Los productos plastiflcantes, anticongelantes, etc., no pueden ser uttlizados si no se ensayan previamente con los mismos áridos, el mismo cemento y en condiciones idénticas a las de la obra.

\section{Ejecuclón del pavimento}

\section{Fabricación del hormigón}

Las hormigoneras deben proporcionar una mezcla intima y regular de los componentes del hormtgón. A este respecto, las hormigoneras de paletas o mezcladoras son preferibles a las hormigoneras de caída libre. En obras de más de $3.000 \mathrm{~m}^{2}$, la dosiflcación de materiales debe efectuarse en peso o por un procedimiento que dé Ia misma precistón. No son admitidas las hormigoneras de una capacidad inferior a los 250 l. Es importante que no se produzca segregación alguna en el stlo de hormigón. Se cumplirá esta condición si el silo es de poca altura, sus paredes son rigidas y si está provisto de una abertura doble. Señalemos, pues, que en suiza se pueden encontrar actualmente fábricas de hormigón bien equipadas, procedentes de obras de grandes presas.

\section{Transporte}

Si no es para los vehículos sin ballestas de suspensión, las normas no fljan límite a la distancia de transporte del hormigón fresco. Especifican, únicamente, que el hormigón debe estar protegido contra las influencias atmosféricas, no debe estar sujeto a segregación y que su puesta en obra debe terminar antes del comienzo del fraguado.

Fig. 3.-Croquis de un silo de bormigón fáeil de lnstalar. Poca altura y $\mathbf{2 , 5} \mathrm{m}^{3}$ de capacladid útil, aproximadamente. Las dos gberturas glargadas
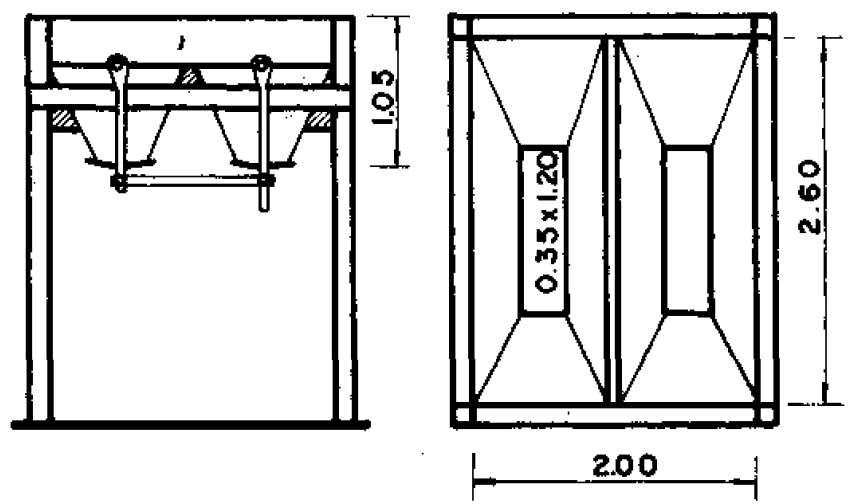

\section{Encofrados}

Deben estar fljados sólldamente sobre una plantilla continua de hormigón o mortero fino, a fin de que no puedan desplazarse ni vertical nf horizontalmente.

\section{Distribución}

En las grandes obras no puede hacerse una distribución económica de hormigón más que por medios mecánicos, los cuales dan, además, una mejor calidad al frme. Los dos tipos de distribuidoras, de cuba o paleta, dan buenos resultados. 


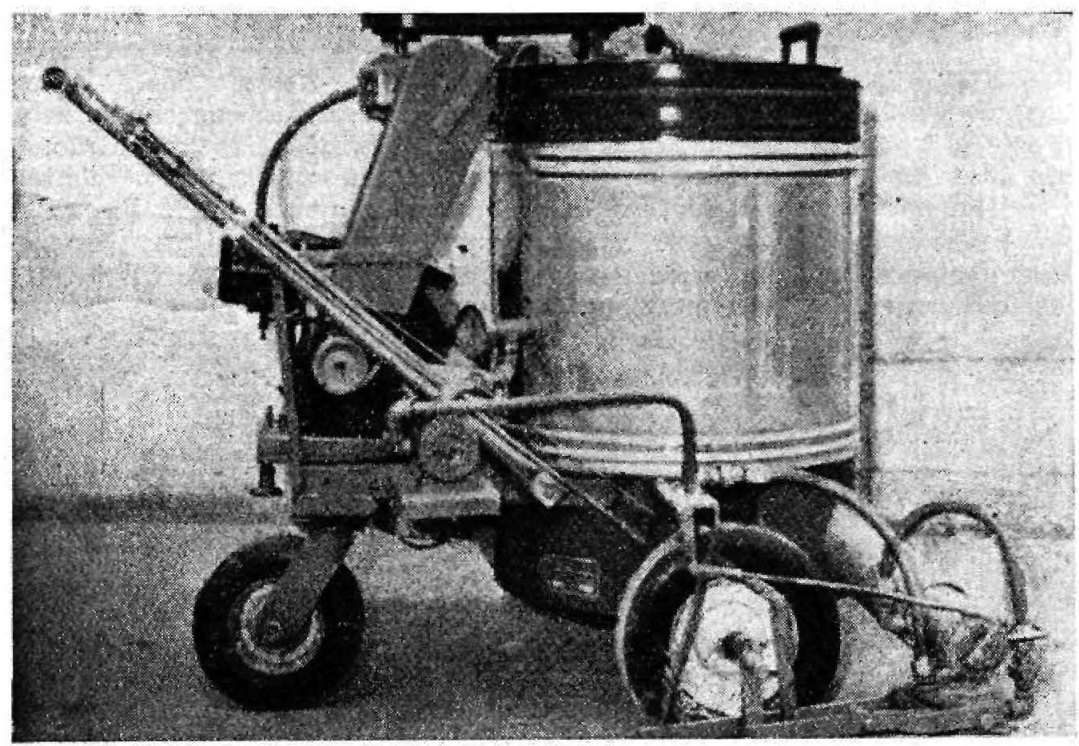

Fig. 4. - Carro vertedor para juntas. Calefacción indirecta, regulador de temperatura, mezela automatica. EI condueto de saliđa está también calentado.

\section{Cerrado}

El hormigón debe ser compactado por medio de máquinas convenientes. Si la maquinaria utilizada es bastante potente, se podrá compactar las dos capas al mismo tiempo. Las normas admiten todos los medios de compactación, sean las vigas vibrantes, las agujas vibrantes o la maquinaria que actúa por apisonado.

\section{Superficie}

Para la terminación de la superflcie, sólo pueden utilizarse máquinas que trabajen sobre el ancho completo de la banda a hormigonar. No se admite añadir mortero o agua para facilitar la terminación. El aspecto y la estructura de la superficie pueden adaptarse a las condiciones locales y nada se ha prescrito a este respecto. Las normas mencionan terminados especiales como fuertes acanaladuras y capas de rodadura de grava dura y hormigón sin arena. Este último tratamiento superficial consiste en un hormigón de alta dosificación compuesto por grava dura 3-6 mm, con muy poca o sin nada de arena.

\section{Obturación de las juntas}

Las prescripciones de ejecución para la obturación de juntas SNV 40470, son totalmente nuevas y se han establecido basándose en numerosos ensayos efectuados en el LFEM y teniendo en cuenta las experiencias de la práctica.

En poco tiempo aparecieron, igualmente, las hojas SNV 71620,71625 y 71627 , relativas a las exigencias de calidad de los materiales de rellenos de juntas tipo KBH (normales) y KTH (resistentes a aceites y esencias minerales).

Estas normas fijan, para las juntas y para el propio firme de hormigón, una duración de garantía de cinco años.

Estas garantías no afectan más que a obras realizadas de acuerdo con las nuevas prescripciones de calidad y de puesta en obra. En obras de alguna importancia, sólo pueden utilizarse los derretidores y carros vertedores con calefacción indirecta por baño de aceite, provistos de reguladores de temperatura y dispositivos mecánicos de mezcla.

Para todas las juntas, la altura del relleno con mástic debe ser, aproximadamente, igual a 1,5 veces la anchura de la junta, pero no inferior a $15 \mathrm{~mm}$. Si el corte es más profundo, debe rectificarse mediante relleno previo con una materia inerte; tras el enfriamiento, la superficie del prodicto de obturación debe ser cóncava. 


\section{Curado de hormigón}

Aparte del curado tradicional por medio de arena húmeda, las nuevas normas autorizan tamblén el uso de líquidos pulverizados formando una capa protectora (productos de curado).

\section{Puesta en circulación}

No podrá abrirse al tráflco antes de catorce días si se utiliza cemento Portland normal, y antes de siete dias si es cemento de altas resistencias iniclales. Si hay razones que obligan a permitir la circulación antes de dichos plazos, será necesarlo comprobar que el hormigón tiene una resistencia, al menos, igual al $80 \%$ de la que se exige a los veintiocho días.

\section{Control de materiales y recepción del pavimento}

\section{Hormigón fresco}

Hay que tomar, por lo menos, dos series de probetas por $10.000 \mathrm{~m}^{2}$ de firme $\mathrm{y}$ por cada calidad de hormigón. Si las resistencias de estas probetas no al canzan los valores requeridos, será determinante la resistencia a compresión de las muestras extrafidas en obra.

\section{Emparrillados de acero}

Se tomará una muestra de la malla por cada 15 toneladas. Esta muestra tendrá, por lo menos, un metro cuadrado de superflcie y se someterá a ensayo.

Fin obras de más de $\mathbf{5 0 . 0 0 0 ~ m , ~ s e r a ́ ~ s u f l c i e n t e ~ u n a ~ m u e s t r a ~ p o r ~ c a d a ~} \mathbf{3 0}$ toneladas de acero recibido.

\section{Productos de preparación y obturación de juntas}

Se realizará un ensayo por $10 \mathrm{t}$ de producto de obturación recibido. Durante las operaciones de relleno de juntas se extraerá, además, en la caldera una muestra por $5.000 \mathrm{~m}$ " de flrme.

\section{Reguloridod de superficie del firme}

Debe ser controlada a $70 \mathrm{~cm}$ del borde de la banda de rotación por medio de una regla de $4 \mathrm{~m}$. Las desigualdades comprobadas con Ia regla deben ser inferiores a $3 \mathrm{~mm}$ en las carreteras nacionales $\mathrm{y}$ principales e inferiores a $5 \mathrm{~mm}$ para las demás carreteras.

\section{Espesores del firme}

Se controla mediante testigos obtentdos a razón de, por lo menos, cuatro unidades cada 10.000 metros cuadrados. 